\title{
ANOTHER METHOD FOR PADMANABHAM'S TRANSFORMATION FORMULA FOR EXTON'S TRIPLE HYPERGEOMETRIC SERIES $X_{8}$
}

\author{
Yong Sup Kim, Aruun Kumar Rathie, and Junesang Choi \\ Abstract. The object of this note is to derive Padmanabham's trans- \\ formation formula for Exton's triple hypergeometric series $X_{8}$ by using \\ a different method from that of Padmanabham's. An interesting special \\ case is also pointed out.
}

\section{Introduction and preliminaries}

In 1982, Exton [3] introduced a set of 20 triple hypergeometric series $X_{1}$ to $X_{20}$ of which we recall here the definition of $X_{8}$ :

$$
\begin{aligned}
& X_{8}(a, b, c ; d, e, f ; x, y, z) \\
= & \sum_{m, n, p=0}^{\infty} \frac{(a)_{2 m+n+p}(b)_{n}(c)_{p} x^{m} y^{n} z^{p}}{(d)_{m}(e)_{n}(f)_{p} m ! n ! p !},
\end{aligned}
$$

where $(\alpha)_{n}$ denotes the Pochhammer symbol defined by

$$
(\alpha)_{n}=\frac{\Gamma(\alpha+n)}{\Gamma(\alpha)} \quad(\alpha \neq 0,-1,-2, \ldots ; n=0,1,2, \ldots) .
$$

The precise three-dimensional region of convergence of (1.1) is given by Srivastava and Karlsson [9, p. 101, Entry 41a]:

$$
4 r=(s+t-1)^{2}, \quad|x|<r, \quad|y|<s, \quad \text { and } \quad|z|<t,
$$

where the positive quantities $r, s$ and $t$ are associated radii of convergence. For details about this function and many other three-variables hypergeometric functions, one refers to Srivastava and Karlsson [9].

Received August 7, 2008.

2000 Mathematics Subject Classification. Primary 33C20, 33C60; Secondary 33C70, $33 \mathrm{C} 65$.

Key words and phrases. triple hypergeometric series $X_{8}$, Horn functions, Laplace integral, Srivastava and Panda's function, Dixon's summation theorem for ${ }_{3} F_{2}(1)$. 
Exton [3] gave the following Laplace integral representation of (1.1):

$$
\begin{aligned}
& X_{8}(a, b, c ; d, e, f ; x, y, z) \\
= & \frac{1}{\Gamma(a)} \int_{0}^{\infty} e^{-u} u_{0}^{a-1} F_{1}\left(-; d ; u^{2} x\right){ }_{1} F_{1}(b ; e ; u y){ }_{1} F_{1}(c ; f ; u z) d u,
\end{aligned}
$$

provided $\Re(a)>0$.

It may be remarked in passing that $X_{8}$ reduces to Horn's function $H_{4}$ when $z \rightarrow 0$ and the Appell's function $F_{2}$ when $x \rightarrow 0$.

Srivastava and Panda [11, p. 423, Eq. (26)] presented a definition of a general double hypergeometric function:

$$
\begin{aligned}
& \left.F_{l: m ; n}^{p: q ; k}\left[\begin{array}{cccc}
\left(a_{p}\right) & :\left(b_{q}\right) & ;\left(c_{k}\right) & ; \\
\left(\alpha_{l}\right) & :\left(\beta_{m}\right) & ;\left(\gamma_{n}\right) & ;
\end{array}\right], y\right] \\
= & \sum_{r, s=0}^{\infty} \frac{\prod_{j=1}^{p}\left(a_{j}\right)_{r+s} \prod_{j=1}^{q}\left(b_{j}\right)_{r} \prod_{j=1}^{k}\left(c_{j}\right)_{s} x^{r} y^{s}}{\prod_{j=1}^{l}\left(\alpha_{j}\right)_{r+s} \prod_{j=1}^{m}\left(\beta_{j}\right)_{r} \prod_{j=1}^{n}\left(\gamma_{j}\right)_{s} r ! s !},
\end{aligned}
$$

where the several cases of convergence conditions are given in [10, p. 64]. Note that Srivastava and Panda's function (1.4) is more general than the one defined by Kampé de Fériet [5] ( $c f$. Appell et Kampé de Fériet [1, p. 150, Eq. (29)].

In 2003, Padmanabham [6] obtained the following result for $X_{8}$ :

$$
\begin{aligned}
& X_{8}(a, b, b ; d, c, c ; x,-x, x) \\
= & F_{0: 3 ; 1}^{2: 2 ; 0}\left[\begin{array}{clll}
\frac{1}{2} a, \frac{1}{2} a+\frac{1}{2} & : b, c-b & ;--- & ; \\
---- & : & c, \frac{1}{2} c, \frac{1}{2} c+\frac{1}{2} & ; d
\end{array}\right]
\end{aligned}
$$

by employing the following result due to Ramanujan [4] involving product of two generalized hypergeometric series:

$$
{ }_{1} F_{1}(a ; b ; x) \times{ }_{1} F_{1}(a ; b ;-x)={ }_{2} F_{3}\left(a, b-a ; b, \frac{1}{2} b, \frac{1}{2} b+\frac{1}{2} ; \frac{x^{2}}{4}\right) .
$$

Consider the special case of (1.5) when $c=2 b$ :

$$
\begin{aligned}
& X_{8}(a, b, b ; d, 2 b, 2 b ; x,-x, x)
\end{aligned}
$$

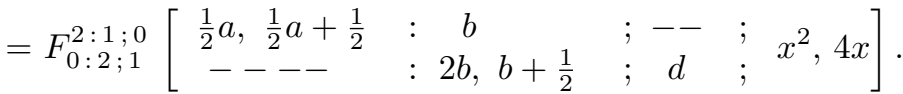

It is interesting here to point out that, instead of (1.6), the identity (1.7) can also be established by using the following well-known Preece's identity [7] involving product of two generalized hypergeometric series:

$$
{ }_{1} F_{1}(a ; 2 a ; x) \times{ }_{1} F_{1}(a ; 2 a ;-x)={ }_{1} F_{2}\left(a ; 2 a, a+\frac{1}{2} ; \frac{x^{2}}{4}\right) .
$$

We will derive Padmanabham's transformation formula (1.5) for Exton's triple hypergeometric series $X_{8}$ by using a different method from that of Padmanabham's. For our purpose, we recall here Dixon's theorem [8, p. 250] for 
the well poised ${ }_{3} F_{2}(1)$ :

$$
\begin{aligned}
&{ }_{3} F_{2}\left[\begin{array}{c}
a, b, c \\
1+a-b, 1+a-c
\end{array} ; 1\right] \\
&= \frac{\Gamma\left(1+\frac{1}{2} a\right) \Gamma(1+a-b) \Gamma(1+a-c) \Gamma\left(1+\frac{1}{2} a-b-c\right)}{\Gamma(1+a) \Gamma\left(1+\frac{1}{2} a-b\right) \Gamma\left(1+\frac{1}{2} a-c\right) \Gamma(1+a-b-c)} \\
&(\Re(a-2 b-2 c)>-2) .
\end{aligned}
$$

We also recall the following well-known identities involving the Pochhammer symbol in (1.2) (see [8, p. 6-8]):

$$
\begin{gathered}
(\alpha)_{n-p}=\frac{(-1)^{p}(\alpha)_{n}}{(1-\alpha-n)_{p}} \quad \text { and } \quad(n-p) !=\frac{(-1)^{p} n !}{(-n)_{p}}(\alpha=1) ; \\
(\alpha)_{2 n}=2^{2 n}\left(\frac{\alpha}{2}\right)_{n}\left(\frac{\alpha}{2}+\frac{1}{2}\right)_{n} ; \\
\frac{\Gamma(\alpha-n)}{\Gamma(\alpha)}=\frac{(-1)^{n}}{(1-\alpha)_{n}} \\
(\alpha)_{m}(\alpha+m)_{n}=(\alpha)_{m+n} .
\end{gathered}
$$

\section{Derivation of (1.5)}

Replacing $c$ by $b, e$ and $f$ by $c, y$ by $-x$, and $z$ by $x$ in (1.1), we have

$$
\begin{aligned}
X_{8} & :=X_{8}(a, b, b ; d, c, c ; x,-x, x) \\
& =\sum_{m=0}^{\infty} \sum_{n=0}^{\infty} \sum_{p=0}^{\infty} \frac{(a)_{2 m+n+p}(b)_{n}(b)_{p}(-1)^{n} x^{m+n+p}}{(d)_{m}(c)_{n}(c)_{p} m ! n ! p !},
\end{aligned}
$$

which, upon using $(a)_{2 m+n+p}=(a)_{2 m}(a+2 m)_{n+p}$, becomes

$$
X_{8}=\sum_{m=0}^{\infty} \frac{(a)_{2 m} x^{m}}{(d)_{m} m !} \sum_{n=0}^{\infty} \sum_{p=0}^{\infty} \frac{(a+2 m)_{n+p}(b)_{n}(b)_{p}(-1)^{n} x^{n+p}}{(c)_{n}(c)_{p} n ! p !}
$$

By making use of the well-known formal manipulation for double series (for more related formulas, see [2]):

$$
\sum_{n=0}^{\infty} \sum_{k=0}^{\infty} A_{k, n}=\sum_{n=0}^{\infty} \sum_{k=0}^{n} A_{k, n-k}
$$

in (2.1), we obtain

$$
X_{8}=\sum_{m=0}^{\infty} \frac{(a)_{2 m} x^{m}}{(d)_{m} m !} \sum_{n=0}^{\infty} \sum_{p=0}^{n} \frac{(a+2 m)_{n}(b)_{n-p}(b)_{p}(-1)^{n-p} x^{n}}{(c)_{p}(c)_{n-p}(n-p) ! p !} .
$$


Applying (1.10) and (1.11) to (2.2), we get

$$
\begin{aligned}
X_{8}= & \sum_{m=0}^{\infty} \frac{\left(\frac{a}{2}\right)_{m}\left(\frac{a}{2}+\frac{1}{2}\right)_{m}(4 x)^{m}}{(d)_{m} m !} \sum_{n=0}^{\infty} \frac{(a+2 m)_{n}(b)_{n}(-x)^{n}}{(c)_{n} n !} \\
& \cdot \sum_{p=0}^{n} \frac{(-n)_{p}(b)_{p}(1-c-n)_{p}}{(1-n-b)_{p}(c)_{p} p !} \\
= & \sum_{m=0}^{\infty} \frac{\left(\frac{a}{2}\right)_{m}\left(\frac{a}{2}+\frac{1}{2}\right)_{m}(4 x)^{m}}{(d)_{m} m !} \sum_{n=0}^{\infty} \frac{(a+2 m)_{n}(b)_{n}(-x)^{n}}{(c)_{n} n !} \\
& \cdot{ }_{3} F_{2}\left[\begin{array}{c}
-n, b, 1-n-c \\
1-n-b, c
\end{array}\right] .
\end{aligned}
$$

Applying Dixon's theorem (1.9) to ${ }_{3} F_{2}(1)$ in $(2.3)$, we obtain

$$
\begin{aligned}
X_{8}= & \sum_{m=0}^{\infty} \frac{\left(\frac{a}{2}\right)_{m}\left(\frac{a}{2}+\frac{1}{2}\right)_{m}(4 x)^{m}}{(d)_{m} m !} \sum_{n=0}^{\infty} \frac{(a+2 m)_{n}(b)_{n}(-x)^{n}}{(c)_{n} n !} \\
& \cdot \mathcal{A}(b, c ; n),
\end{aligned}
$$

where, for convenience,

$$
\mathcal{A}(b, c ; n):=\frac{\Gamma(c) \Gamma(1-b-n) \Gamma\left(c-b+\frac{1}{2} n\right) \Gamma\left(1-\frac{1}{2} n\right)}{\Gamma(c-b) \Gamma\left(c+\frac{1}{2} n\right) \Gamma\left(1-b-\frac{1}{2} n\right) \Gamma(1-n)} .
$$

By making use of Legendre's duplication formula for the Gamma function:

$$
\Gamma\left(\frac{1}{2}\right) \Gamma(2 \alpha)=2^{2 \alpha-1} \Gamma(\alpha) \Gamma\left(\alpha+\frac{1}{2}\right),
$$

we have

$$
\mathcal{A}(b, c ; n)=\frac{\Gamma(c) \Gamma(1-b-n) \Gamma\left(c-b+\frac{1}{2} n\right)}{\Gamma(c-b) \Gamma\left(c+\frac{1}{2} n\right) \Gamma\left(1-b-\frac{1}{2} n\right)} \cdot \frac{2^{n} \Gamma\left(\frac{1}{2}\right)}{\Gamma\left(\frac{1}{2}-\frac{1}{2} n\right)},
$$

from which we see that

$$
\mathcal{A}(b, c ; n)=0
$$

whenever $n$ is an odd positive integer.

Considering (2.5), we can rewrite $X_{8}$ in (2.4) as follows:

$$
\begin{aligned}
X_{8}= & \sum_{m=0}^{\infty} \frac{\left(\frac{a}{2}\right)_{m}\left(\frac{a}{2}+\frac{1}{2}\right)_{m}(4 x)^{m}}{(d)_{m} m !} \sum_{n=0}^{\infty} \frac{(a+2 m)_{2 n}(b)_{2 n} x^{2 n}}{(c)_{2 n}(2 n) !} \\
& \cdot \frac{\Gamma(c) \Gamma(1-b-2 n) \Gamma(c-b+n)}{\Gamma(c-b) \Gamma(c+n) \Gamma(1-b-n)} \cdot \frac{2^{2 n} \Gamma\left(\frac{1}{2}\right)}{\Gamma\left(\frac{1}{2}-n\right)} .
\end{aligned}
$$

If we employ (1.11), (1.12), and (1.13) in (2.6), we finally obtain

$$
X_{8}=\sum_{n=0}^{\infty} \sum_{m=0}^{\infty} \frac{\left(\frac{1}{2} a\right)_{m+n}\left(\frac{1}{2} a+\frac{1}{2}\right)_{m+n}(b)_{n}(c-b)_{n} x^{2 n}(4 x)^{m}}{(c)_{n}\left(\frac{1}{2} c\right)_{n}\left(\frac{1}{2} c+\frac{1}{2}\right)_{n}(d)_{m} n ! m !}
$$


which, upon using (1.4), leads to the desired formula (1.5).

\section{References}

[1] P. Appell et J. Kampé de Fériet, Fonctions Hypergéométriques et Hypersphériques Polynomes D'Hermite, Gauthier-Villars, Paris, 1926.

[2] J. Choi, Notes on formal manipulations of double series, Commun. Korean Math. Soc. 18 (2003), no. 4, 781-789.

[3] H. Exton, Hypergeometric function of three variables, J. Indian Acad. Maths. 4 (1982), no. 2, 113-119.

[4] G. H. Hardy, A chapter from Ramanujan's notebook, Proc. Cambridge Philos. Soc. 21 (1923), 492-503.

[5] J. Kampé de Fériet, Les fonctions hypergéométriques d'ordre supérieur à deux variables, C. R. Acad. Sci. Paris 173 (1921), 401-404.

[6] P. A. Padmanabham, Expansions for a multiple hypergeometric function, Ganita 54 (2003), no. 1, 17-20

[7] C. T. Preece, The product of two generalized hypergeometric functions, Proc. London Math. Soc. 22 (1924), 370-380.

[8] H. M. Srivastava and J. Choi, Series Associated with the Zeta and Related Functions, Kluwer Academic Publishers, Dordrecht, Boston, and London, 2001.

[9] H. M. Srivastava and P. W. Karlsson, Multiple Gaussian Hypergeometric Series, Halsted Press (Ellis Horwood Limited, Chichester); Wiley, New York, Chichester, Brisbane, and Toronto, 1985.

[10] H. M. Srivastava and H. L. Manocha, A Treatise on Generating Functions, Halsted Press (Ellis Horwood Limited, Chichester); Wiley, New York, Chichester, Brisbane, and Toronto, 1984.

[11] H. M. Srivastava and R. Panda, An integral representation for the product of two Jacobi polynomials, J. London Math. Soc. 12 (1976), no. 2, 419-425.

YONG SUP KIM

Department of Mathematics Education

WONKWANG UNIVERSITY

IKSAN 570-749, KoREA

E-mail address: yspkim@wonkwang.ac.kr

Aruun Kumar Rathie

Department of Mathematics

Vedant College of Engineering and Technology

Tulsi-323021, Dist. Bundi Rajasthan State, India

E-mail address: akrathie@rediffmail.com

JunESANG CHOI

Department of Mathematics Education

DONGGUK UNIVERSITY

KYONGJU 780-714, KoreA

E-mail address: junesang@mail.dongguk.ac.kr 